\title{
Effect Of Educational Nutritional Program For Pregnant Women On Fetal Out Come At Birth.
}

\author{
* Prof. Dr. Sonia Gamal Elsharkawy, **Prof. Dr. Hayat Imam Mohamed, \\ ***Dr.Mervat Gaber Zaghloul,Nor Elhouda Mohammed Elsayed. \\ *Prof and Head of Pediatric Department, Faculty of Medicine-Suez Canal \\ University, ** Prof. of Maternal and Newborn Health Nursing, Faculty of Nursing- \\ Cairo University, *** Lecturer of Maternity, Obstetric and Gynecological Nursing, \\ Faculty of Nursing - Port said University.
}

\begin{abstract}
Objectives: The aim of this study is to evaluate the impact of a designed educational nutrition program for primipara on neonatal weight.

Subject and Methods: The study was carried out at El-Tadamon hospital in PortSaid City This is a private hospital providing outpatient and inpatient obstetric and gynecological services in using a quasi-experimental (pre \& post test) design The subjects of the study were any primiparous pregnant woman attending the mentioned study setting during the time of the study was eligible for inclusion in the study sample if she fulfills the following eligibility criteria.
\end{abstract}

Results: The results revealed that there was a significant improvement in the woman knowledge score about nutrition during pregnancy before and after program .

Conclusion: Educational program has a significant and improvement impact on knowledge regarding nutrition during pregnancy, and this is negatively reflected on their dietary practices and habits

Key words: Effect of Educational Nutritional program for Pregnancy and birth outcome. 


\section{INTRODUCTION}

Pregnancy is one of the most important periods in the life of a woman, a family and a society. Extraordinary attention is therefore given to antenatal care by the health care systems of most countries. The goal of antenatal care is to prevent health problems in both infant and mother and to see that each newborn child has a good start.

The care provided needs to be appropriate and not excessive. New technologies need to be implemented continually, while older services need to be reconsidered. The care for each pregnant woman needs to be individualized based on her own needs and wish (Gabbe et al., 2007).

A pregnant woman should be helped to understand the importance of good nutrition for herself and for her fetus, she may be more motivated than at other times in her life to improve her dietary habits. She should also be encouraged to continue her new interest in nutrition after the baby arrives (Macintosh and Mack, 2000)

Antenatal care is a systematic and comprehensive physical, social and psychological care of the pregnant woman to maintain, protect and promote the health for mothers and her fetus, and to help a woman approach pregnancy and birth as positive experiences. To a large extent antenatal care can contribute greatly to this purpose. Many studies have shown the importance of the antenatal care and its effect on reducing the incidence of maternal and infant morbidity, mortality and improving pregnancy outcome (Taylor et al., 2001).

Maternal malnutrition prior to and during pregnancy manifested by low bodyweight, short stature, Inadequate energy intake during pregnancy and coexisting micronutrient deficiency are considered major determinants in developing countries where the burden is too high. Low Birth Weight (LBW) is a multi-factorial outcome and its prevention requires a lifecycle approach and interventions must be continued for several generations. So, most interventions are now targeted during pregnancy primarily due to the increased nutritional demand and aggravations of already existing inadequacy in most women (Alam, 2009).

Dietary counseling should be an ongoing aspect of prenatal care;

it is not enough to talk about it at the first visit. It includes information on weight, dietary assessment, and dietary planning to prevent complications and problems for the pregnant women and their babies during pregnancy, labor, and post partum period (Gabbe et al., 2007).

\section{AIM OF STUDY:}

The aim of this study is to evaluate the impact of a designed educational nutrition program for primipara on neonatal weight.

\section{SUBJECT AND METHODS:}

This study was carried out using a controlled quasi-experimental research design with pre-post assessment. The study was carried out at El-Tadamon hospital in Port-Said city. This is a private hospital providing outpatient and inpatient obstetrical and gynecological services. the process of data collection and implementation of the 
educational program consumed 15 months from April 2011 to September 2012 The data were collected according to One tool was developed and used by the researcher for data collection.

\section{Ethical Considerations}

An informed consent form was signed by each participant before collecting any data. The form explained the study aim in a simple and clear manner to be understood by common people. No harmful maneuvers were performed or used, and no foreseen hazards were anticipated from conducting the study. Participants were informed about their right to withdraw from the study at any time without giving any reason. Data were considered confidential and not be used outside this study without approval..

\section{Content Validity:}

The researcher designed an opinnionnaire sheet to test the content validity of the assessment questionnaire sheet for nurses and mothers by a jury including 10 Experts in the field of obstetric and gynecology from medical and nursing faculty staff. It involved two parts:

C-The opinions of the experts for each item were recorded on a two point scale: relevant, not relevant and clear, not clear.

D-General or overall opinion about the form, they were requested to express their opinions and comments on the tool and provide any suggestions for any additional or omissions of items. Then necessary modifications were done. This phase was carried out in a period of two months.

A pilot study, which was carried out on $10 \%$ from pregnant woman .The main purpose of the pilot study were to test the clarity, feasibility of the tools and whether it was understandable, and to determine the time needed to fill the tool. The tool was handed to participants to fill it and collected by researcher. The time for the completion of the questionnaire sheet was ranged from 30 to 60 minutes. Then reliability of the tool was done which lead to omission. Following this pilot study, the process of data collection and implementation of educational program consumed 15 months from April 2011 to September 2012.

\section{RESULTS}

Table (1) shows that women in study and control groups had a close mean age (27.7 and 28.0 years respectively). The highest percentages had intermediate education (46.4\% and 58.9\%, respectively). More than $80 \%$ of them were housewives, with crowding index less than two persons per room.

As regards their husbands, the table demonstrates a higher mean age in the study group was (29.9 years) compared to the control group (31.5 years) and this was the only difference of statistical significance between the two groups $(p=0.02)$. More than two-thirds of the husbands were employees and more than half of them had intermediate education.

Figure 1 indicates a higher prevalence of consanguinity among women in the control group (43.8\%), compared to the study group (35.7\%). However, the difference was not statistically 


\section{ignificant $(\mathrm{p}=0.22)$.}

Concerning women's dietary intake before the intervention, Table 2 indicates that the women in the control group had statistically significantly higher daily intake of vegetables and fruits $(\mathrm{p}=0.01)$, dairies $(\mathrm{p}=0.048)$, and fats $(\mathrm{p}<0.001)$. Meanwhile, women in the study group had higher intake of proteins $(p=0.004)$. However, the dietary intake according to Recommended Daily Allowances (RDA) was very low in both groups for the main food groups. Thus, only six (5.4\%) women in the study group had balanced diet, compared to only one $(0.9 \%)$ in the control group, but the difference was not statistically significant $(\mathrm{p}=0.12)$.

Tale 3 demonstrates statistically significant improvements in the postintervention dietary knowledge of women in the study group in all tested elements ( $\mathrm{p}<0.001$ ). In total, $27.7 \%$ of the women had satisfactory knowledge at pre the intervention phase, and this increased to $95.5 \%$ at the posttest $(\mathrm{p}<0.001)$. No statistically significant differences could be revealed in control group women's knowledge between pre and post intervention tests. In total, $17.9 \%$ of the women had satisfactory knowledge at pre the intervention phase, and this slightly increased to $18.8 \%$ at the posttest with no statistical significance $(\mathrm{p}=0.86)$.

Concerning the newborn outcomes Table 4 points to statistically significant differences in the respiratory rate $(\mathrm{p}=0.007)$, and heart rate $(\mathrm{p}<0.001)$. As noticed from the table, more newborns in the study group had abnormal respiratory rate, but more newborns in the control group had abnormal heart rates. The mean body temperature in the two groups was similar.

Table 5 compares the newborn anthropometric measurements in the study and control groups. It indicates statistically significant differences in the body weight and head circumference $(\mathrm{P}<0.001)$. It is clear that the newborns in the study group had higher mean body weight and head circumference. Meanwhile, the length and chest circumferences did not show statistically significant differences.

Comparison of the newborn physical assessment findings in the study and control groups (Table 6) demonstrated that newborns in the study group had statistically significantly more Moro reflex $(\mathrm{p}=0.001)$, and less scalp molding $(\mathrm{p}=0.005)$ and caput succedaneum $(\mathrm{p}<0.001)$. They had higher percentages of equal size of the eyes $(\mathrm{p}<0.001)$, less nasal obstruction $(\mathrm{p}<0.001)$ and asymmetric chest $(\mathrm{p}<0.001)$. On the other hand, newborns in the control group had statistically significantly more abnormal movement in the extremities $(p<0.001)$, firm/distended abdomen $(p<0.001)$, imperforate anus $(\mathrm{p}<0.001)$, and less equal breath sounds $(\mathrm{p}<0.001)$.

Table 7 shows that a statistically significantly higher percentage of newborns in the control group had their Apgar score $<7(\mathrm{p}<0.001)$. They also had a higher rate of NICU admission ( $\mathrm{p}<0.001)$. 
Table 8 presents the relations between the intake of balanced diet after intervention among women in the study group and their personal characteristics. As the table indicates, no statistically significant associations could be revealed with any of the women's characteristics.

Table (1): Socio-demographic characteristics of women in the study and control groups

\begin{tabular}{|c|c|c|c|c|c|c|}
\hline & \multicolumn{4}{|c|}{ TGroup } & \multirow{3}{*}{$\begin{array}{l}\mathrm{X}^{2} \\
\text { Test }\end{array}$} & \multirow{3}{*}{ p-value } \\
\hline & \multicolumn{2}{|c|}{$\begin{array}{l}\text { Study } \\
(n=112)\end{array}$} & \multicolumn{2}{|c|}{$\begin{array}{l}\text { Control } \\
(n=112)\end{array}$} & & \\
\hline & No. & $\%$ & No. & $\%$ & & \\
\hline $\begin{array}{l}\text { Age (years): } \\
<25 \\
25- \\
30-35 \\
\text { Range } \\
\text { Mean } \pm \text { SD }\end{array}$ & $\begin{array}{l}30 \\
45 \\
37 \\
19.0 \\
27.7\end{array}$ & $\begin{array}{l}26.8 \\
40.2 \\
33.0 \\
5.0 \\
.2\end{array}$ & $\begin{array}{l}23 \\
50 \\
39 \\
19.0 \\
28.0\end{array}$ & $\begin{array}{l}20.5 \\
44.6 \\
34.8 \\
5.0 \\
4.2\end{array}$ & 1.24 & 0.54 \\
\hline $\begin{array}{l}\text { Education: } \\
\text { Illiterate } \\
\text { Read/write } \\
\text { Basic } \\
\text { Intermediate } \\
\text { University } \\
\text { Higher }\end{array}$ & $\begin{array}{l}4 \\
6 \\
11 \\
52 \\
26 \\
13\end{array}$ & $\begin{array}{l}3.6 \\
5.4 \\
9.8 \\
46.4 \\
23.2 \\
11.6\end{array}$ & $\begin{array}{l}3 \\
3 \\
6 \\
66 \\
20 \\
14\end{array}$ & $\begin{array}{l}2.7 \\
2.7 \\
5.4 \\
58.9 \\
17.9 \\
12.5\end{array}$ & 5.09 & 0.40 \\
\hline $\begin{array}{l}\text { Job: } \\
\text { Housewife } \\
\text { Working } \\
\end{array}$ & $\begin{array}{l}22 \\
90 \\
\end{array}$ & $\begin{array}{l}19.6 \\
80.4 \\
\end{array}$ & $\begin{array}{l}17 \\
95 \\
\end{array}$ & $\begin{array}{l}15.2 \\
84.8 \\
\end{array}$ & 0.78 & 0.38 \\
\hline $\begin{array}{l}\text { Crowding index: } \\
<2 \\
2+\end{array}$ & $\begin{array}{l}91 \\
21 \\
\end{array}$ & $\begin{array}{l}81.3 \\
18.8 \\
\end{array}$ & $\begin{array}{l}95 \\
17 \\
\end{array}$ & $\begin{array}{l}84.8 \\
15.2 \\
\end{array}$ & 0.51 & 0.48 \\
\hline $\begin{array}{l}\text { Husband age (years): } \\
<30 \\
30- \\
35+ \\
\text { Range } \\
\text { Mean } \pm \text { SD }\end{array}$ & $\begin{array}{l}57 \\
29 \\
26 \\
20.0 \\
29.9\end{array}$ & $\begin{array}{l}50.9 \\
25.9 \\
23.2 \\
3.0 \\
.8\end{array}$ & $\begin{array}{l}37 \\
34 \\
41 \\
22.0 \\
31.5\end{array}$ & $\begin{array}{l}33.0 \\
30.4 \\
36.6 \\
0.0 \\
4.6\end{array}$ & 8.01 & $0.02 *$ \\
\hline $\begin{array}{l}\text { Husband job: } \\
\text { Employee } \\
\text { Worker }\end{array}$ & $\begin{array}{l}78 \\
34 \\
\end{array}$ & $\begin{array}{l}69.6 \\
30.4 \\
\end{array}$ & $\begin{array}{l}75 \\
37 \\
\end{array}$ & $\begin{array}{l}67.0 \\
33.0 \\
\end{array}$ & 0.19 & 0.67 \\
\hline $\begin{array}{l}\text { Husband education: } \\
\text { Illiterate } \\
\text { Read/write } \\
\text { Basic } \\
\text { Intermediate } \\
\text { University } \\
\text { Higher }\end{array}$ & $\begin{array}{l}0 \\
6 \\
11 \\
61 \\
27 \\
7\end{array}$ & $\begin{array}{l}0.0 \\
5.4 \\
9.8 \\
54.5 \\
24.1 \\
6.3 \\
\end{array}$ & $\begin{array}{l}1 \\
4 \\
8 \\
60 \\
32 \\
7\end{array}$ & $\begin{array}{l}0.9 \\
3.6 \\
7.1 \\
53.6 \\
28.6 \\
6.3 \\
\end{array}$ & 2.31 & 0.81 \\
\hline
\end{tabular}

(*) Statistically significant at $p<0.05$

Figure (1): Comparison of consanguinity among women in the study and control groups 


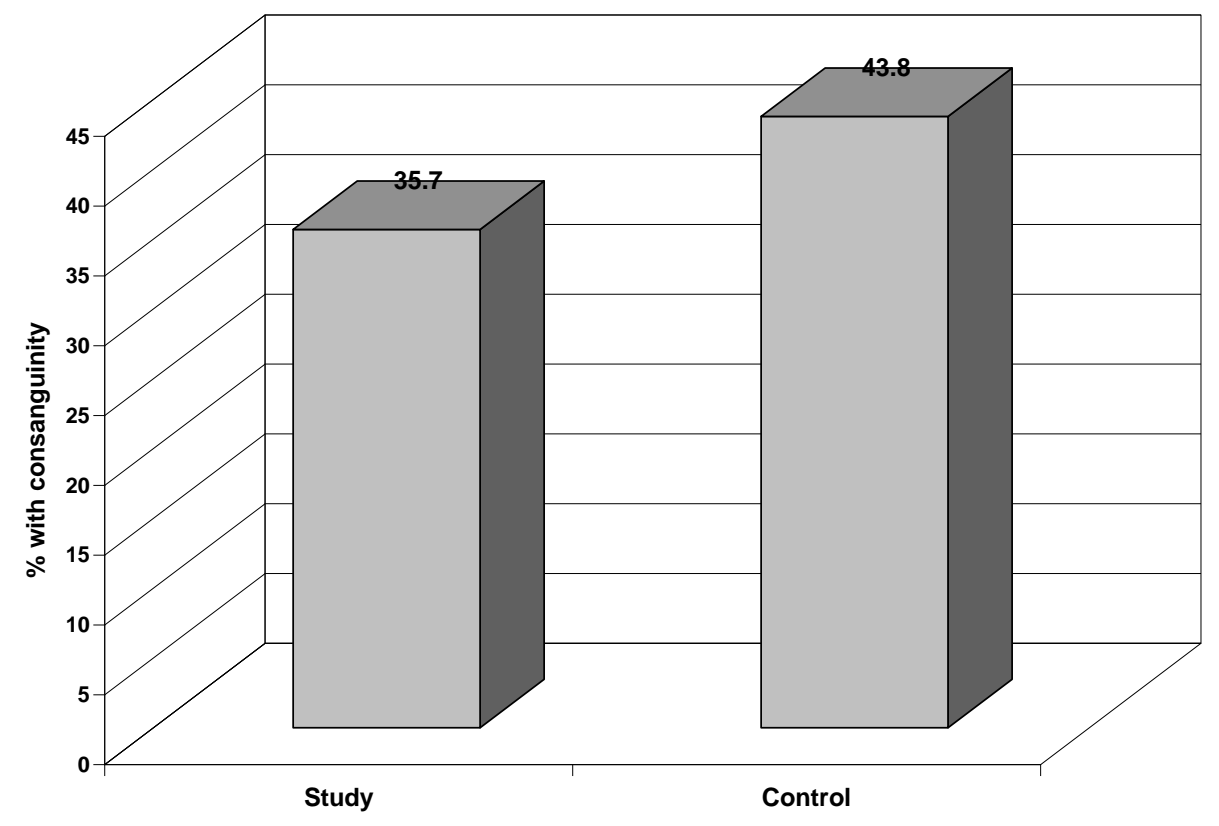


Table (2) Pre-intervention dietary intakes during pregnancy among women in the study and control groups

\begin{tabular}{|c|c|c|c|c|c|c|}
\hline \multirow{3}{*}{ Daily intake of: } & \multicolumn{4}{|l|}{ Group } & \multirow{3}{*}{$\begin{array}{l}\text { Mann } \\
\text { Whitney } \\
\text { Test }\end{array}$} & \multirow{3}{*}{ p-value } \\
\hline & \multicolumn{2}{|c|}{\begin{tabular}{|l} 
Study \\
$(n=112)$
\end{tabular}} & \multicolumn{2}{|c|}{\begin{tabular}{|l} 
Control \\
$(\mathrm{n}=112)$
\end{tabular}} & & \\
\hline & No. & $\%$ & \begin{tabular}{|l|} 
No. \\
\end{tabular} & $\%$ & & \\
\hline $\begin{array}{l}\text { Cereals: } \\
\text { Range } \\
\text { Mean } \pm \text { SD }\end{array}$ & \multicolumn{2}{|l|}{$\begin{array}{l}1.0-4.0 \\
2.1 \pm 0.8\end{array}$} & \multicolumn{2}{|l|}{$\begin{array}{l}1.0-4.0 \\
2.2 \pm 0.6\end{array}$} & 2.26 & 0.13 \\
\hline \begin{tabular}{|l|} 
Proteins: \\
Range \\
Mean \pm SD
\end{tabular} & \multicolumn{2}{|c|}{$\begin{array}{l}0.0-3.0 \\
1.3 \pm 0.5\end{array}$} & \multicolumn{2}{|l|}{$\begin{array}{l}0.0-2.0 \\
1.1 \pm 0.6\end{array}$} & 8.10 & $0.004 *$ \\
\hline \begin{tabular}{|l|} 
Legumes \\
Range \\
Mean $\pm S D$
\end{tabular} & \multicolumn{2}{|c|}{$\begin{array}{l}0.0-0.2 \\
0.2 \pm 0.5\end{array}$} & \multicolumn{2}{|l|}{$\begin{array}{l}0.0-1.0 \\
0.3 \pm 0.4\end{array}$} & 1.39 & 0.24 \\
\hline $\begin{array}{l}\text { Vegetables/fruits: } \\
\text { Range } \\
\text { Mean } \pm \text { SD }\end{array}$ & \multicolumn{2}{|c|}{$\begin{array}{l}0.0-2.0 \\
0.4 \pm 0.5\end{array}$} & \multicolumn{2}{|l|}{$\begin{array}{l}0.0-2.0 \\
0.6 \pm 0.6\end{array}$} & 6.46 & $0.01 *$ \\
\hline $\begin{array}{l}\text { Dairies: } \\
\text { Range } \\
\text { Mean } \pm \text { SD }\end{array}$ & \multicolumn{2}{|c|}{$\begin{array}{l}0.0-3.0 \\
1.1 \pm 0.7\end{array}$} & \multicolumn{2}{|l|}{$\begin{array}{l}0.0-3.0 \\
1.2 \pm 0.7\end{array}$} & 3.90 & $0.048 *$ \\
\hline $\begin{array}{l}\text { Fats: } \\
\text { Range } \\
\text { Mean } \pm S D\end{array}$ & \multicolumn{2}{|c|}{$\begin{array}{l}0.0-1.0 \\
0.2 \pm 0.4\end{array}$} & \multicolumn{2}{|l|}{$\begin{array}{l}0.0-1.0 \\
0.5 \pm 0.5\end{array}$} & 16.78 & $<0.001 *$ \\
\hline $\begin{array}{l}\text { Sugars: } \\
\text { Range } \\
\text { Mean } \pm S D\end{array}$ & \multicolumn{2}{|c|}{$\begin{array}{l}0.0-4.0 \\
0.7 \pm 0.8\end{array}$} & \multicolumn{2}{|l|}{$\begin{array}{l}0.0-2.0 \\
0.6 \pm 0.6\end{array}$} & 0.03 & 0.86 \\
\hline $\begin{array}{l}\text { Intake according to } \\
\text { recommended daily allowances } \\
\text { (RDA): } \\
\text { Cereals } \\
\text { Proteins } \\
\text { Vegetables/fruits } \\
\text { Dairies } \\
\text { Balanced diet }\end{array}$ & $\begin{array}{l}3 \\
3 \\
1 \\
0 \\
6\end{array}$ & $\begin{array}{l}2.7 \\
2.7 \\
0.9 \\
0.0 \\
5.4\end{array}$ & $\begin{array}{l}1 \\
0 \\
0 \\
0 \\
1\end{array}$ & $\begin{array}{l}0.9 \\
0.0 \\
0.0 \\
0.0 \\
0.9\end{array}$ & $\begin{array}{l}\text { Fisher } \\
\text { Fisher } \\
\text { Fisher } \\
0.00 \\
\text { Fisher }\end{array}$ & $\begin{array}{l}0.62 \\
0.25 \\
1.00 \\
1.00 \\
0.12\end{array}$ \\
\hline
\end{tabular}

(*) Statistically significant at $p<0.05$ 
Table (3): Pre-post-intervention dietary knowledge among women in the study and control groups

\begin{tabular}{|c|c|c|c|c|c|c|}
\hline \multirow{3}{*}{ Satisfactory knowledge $(50 \%+)$ : } & \multicolumn{4}{|c|}{ Time } & \multirow{3}{*}{$\begin{array}{l}\mathbf{X}^{2} \\
\text { Test }\end{array}$} & \multirow{3}{*}{ p-value } \\
\hline & \multicolumn{2}{|c|}{ Pre } & \multicolumn{2}{|c|}{ Post } & & \\
\hline & No. & $\%$ & No. & $\%$ & & \\
\hline Study: & & & & & & \\
\hline Balanced diet & 68 & 60.7 & 110 & 98.2 & 48.26 & $<0.001 *$ \\
\hline Proteins & 43 & 38.4 & 110 & 98.2 & 92.57 & $<0.001 *$ \\
\hline Iron & 41 & 36.6 & 110 & 98.2 & 96.75 & $<0.001 *$ \\
\hline Vitamins & 35 & 31.3 & 108 & 96.4 & 103.06 & $<0.001 *$ \\
\hline Other minerals & 36 & 32.1 & 103 & 92.0 & 85.11 & $<0.001 *$ \\
\hline Fats & 40 & 35.7 & 107 & 95.5 & 88.84 & $<0.001 *$ \\
\hline Fluids & 46 & 41.1 & 108 & 96.4 & 79.88 & $<0.001 *$ \\
\hline Total knowledge: & & & & & & \\
\hline Satisfactory $(50 \%+)$ & 31 & 27.7 & 107 & 95.5 & & \\
\hline Unsatisfactory $(<50 \%)$ & 81 & 72.3 & 5 & 4.5 & 109.02 & $<0.001 *$ \\
\hline Control: & & & & & & \\
\hline Balanced diet & 81 & 72.3 & 84 & 75.0 & 0.21 & 0.65 \\
\hline Proteins & 63 & 56.3 & 63 & 56.3 & 0.00 & 1.00 \\
\hline Iron & 49 & 43.8 & 54 & 48.2 & 0.45 & 0.50 \\
\hline Vitamins & 21 & 18.8 & 21 & 18.8 & 0.00 & 1.00 \\
\hline Other minerals & 11 & 9.8 & 11 & 9.8 & 0.00 & 1.00 \\
\hline Fats & 20 & 17.9 & 23 & 20.5 & 0.26 & 0.61 \\
\hline Fluids & 38 & 33.9 & 41 & 36.6 & 0.18 & 0.67 \\
\hline Total knowledge: & & & & & & \\
\hline Satisfactory $(50 \%+)$ & 20 & 17.9 & 21 & 18.8 & & \\
\hline Unsatisfactory $(<50 \%)$ & 92 & 82.1 & 91 & 81.3 & 0.03 & 0.86 \\
\hline
\end{tabular}

(*) Statistically significant at $p<0.05$

Table (4): Newborn vital signs in the study and control groups at birth

\begin{tabular}{|c|c|c|c|c|c|c|}
\hline & \multicolumn{4}{|c|}{ Group } & \multirow{3}{*}{$\begin{array}{l}X^{2} \\
\text { Test }\end{array}$} & \multirow{3}{*}{ p-value } \\
\hline & \multicolumn{2}{|c|}{$\begin{array}{l}\begin{array}{l}\text { Study } \\
\text { (n=112) }\end{array} \\
\end{array}$} & \multicolumn{2}{|c|}{$\begin{array}{l}\text { Control } \\
(\mathrm{n}=112)\end{array}$} & & \\
\hline & No. & $\%$ & No. & $\%$ & & \\
\hline $\begin{array}{l}\text { Respiratory rate: } \\
\text { Normal } \\
\text { Abnormal } \\
\text { Range } \\
\text { Mean } \pm \text { SD }\end{array}$ & $\begin{array}{l}95 \\
17 \\
20- \\
46 .\end{array}$ & $\begin{array}{l}84.8 \\
15.2 \\
5.5\end{array}$ & $\begin{array}{l}107 \\
5 \\
40-6 \\
47.0\end{array}$ & $\begin{array}{l}95.5 \\
4.5 \\
.3\end{array}$ & 7.26 & $0.007 *$ \\
\hline $\begin{array}{l}\text { Heart rate }(\mathbf{B P M}): \\
<120 \\
120- \\
160+ \\
\text { Range } \\
\text { Mean } \pm \text { SD }\end{array}$ & $\begin{array}{l}2 \\
105 \\
5 \\
110 \\
130 \\
\end{array}$ & $\begin{array}{l}1.8 \\
93.8 \\
4.5 \\
70 \\
+10.4 \\
\end{array}$ & $\begin{array}{l}0 \\
51 \\
61 \\
120 \\
152 .\end{array}$ & $\begin{array}{l}0.0 \\
45.5 \\
54.5 \\
0 \\
15.3 \\
\end{array}$ & 68.21 & $<0.001 *$ \\
\hline $\begin{array}{l}\text { Body temperature: } \\
<37 \\
37- \\
37.5+ \\
\text { Range } \\
\text { Mean } \pm \text { SD }\end{array}$ & $\begin{array}{l}55 \\
56 \\
1 \\
36 . \\
36.5\end{array}$ & $\begin{array}{l}49.1 \\
50.0 \\
0.9 \\
8.0 \\
0.5\end{array}$ & $\begin{array}{l}58 \\
50 \\
4 \\
36.0 \\
36.5\end{array}$ & $\begin{array}{l}51.8 \\
44.6 \\
3.6 \\
9.0 \\
.6\end{array}$ & -- & -- \\
\hline
\end{tabular}


Table (5): Newborn anthropometric measurements in the study and control groups at birth

\begin{tabular}{|c|c|c|c|c|c|c|}
\hline & \multicolumn{4}{|c|}{ Group } & \multirow{3}{*}{$\begin{array}{l}\text { Mann } \\
\text { Whitney } \\
\text { Test }\end{array}$} & \multirow{3}{*}{ p-value } \\
\hline & \multicolumn{2}{|c|}{$\begin{array}{l}\text { Study } \\
(n=112)\end{array}$} & \multicolumn{2}{|c|}{$\begin{array}{l}\text { Control } \\
(n=112)\end{array}$} & & \\
\hline & No. & $\%$ & No. & $\%$ & & \\
\hline $\begin{array}{l}\text { Body weight (gm): } \\
<2500 \\
2500- \\
3000+ \\
\text { Range } \\
\text { Mean } \pm \text { SD }\end{array}$ & $\begin{array}{l}8 \\
8 \\
96 \\
2200 \\
3571\end{array}$ & $\begin{array}{l}7.1 \\
7.1 \\
85.7 \\
500 \\
\pm 562.2\end{array}$ & $\begin{array}{l}3 \\
33 \\
76 \\
2250 \\
3185\end{array}$ & $\begin{array}{l}2.7 \\
29.5 \\
67.9 \\
500 \\
\pm 507.2\end{array}$ & 26.47 & $<0.001^{*}$ \\
\hline $\begin{array}{l}\text { Length }(\mathbf{c m}): \\
<50 \\
50+ \\
\text { Range } \\
\text { Mean } \pm \text { SD } \\
\end{array}$ & $\begin{array}{l}68 \\
44 \\
42.0 \\
48.6 \\
\end{array}$ & $\begin{array}{l}60.7 \\
39.3 \\
.0 \\
5 \\
\end{array}$ & $\begin{array}{l}73 \\
39 \\
38.0 \\
48.3 \\
\end{array}$ & $\begin{array}{l}65.2 \\
34.8 \\
0 \\
0 \\
\end{array}$ & 3.06 & 0.08 \\
\hline $\begin{array}{l}\text { Head circumference }(\mathbf{c m}) \text { : } \\
<35 \\
35+ \\
\text { Range } \\
\text { Mean } \pm \text { SD }\end{array}$ & $\begin{array}{l}94 \\
18 \\
31.0 \\
33.6\end{array}$ & $\begin{array}{l}83.9 \\
16.1 \\
.0 \\
2\end{array}$ & $\begin{array}{l}94 \\
18 \\
30.0 \\
33.3\end{array}$ & $\begin{array}{l}83.9 \\
16.1 \\
.0 \\
9\end{array}$ & 11.88 & $<0.001^{*}$ \\
\hline $\begin{array}{l}\text { Chest circumference }(\mathbf{c m}) \text { : } \\
<35 \\
35+ \\
\text { Range } \\
\text { Mean } \pm \text { SD }\end{array}$ & $\begin{array}{l}112 \\
0 \\
30.0 \\
31.8\end{array}$ & $\begin{array}{l}100.0 \\
0.0 \\
.0 \\
0\end{array}$ & $\begin{array}{l}110 \\
2 \\
30.0 \\
31.8\end{array}$ & $\begin{array}{l}98.2 \\
1.8 \\
.0 \\
9\end{array}$ & Fisher & 0.50 \\
\hline
\end{tabular}

(*) Statistically significant at $p<0.05$ 
Table (6): Newborn physical assessment findings in the study and control groups

\begin{tabular}{|c|c|c|c|c|c|c|}
\hline & \multicolumn{4}{|c|}{ Group } & \multirow{3}{*}{$\begin{array}{l}X^{2} \\
\text { Test }\end{array}$} & \multirow{3}{*}{ p-value } \\
\hline & \multicolumn{2}{|c|}{$\begin{array}{l}\text { Study } \\
(n=112)\end{array}$} & \multicolumn{2}{|c|}{$\begin{array}{l}\text { Control } \\
(\mathrm{n}=112)\end{array}$} & & \\
\hline & No. & $\%$ & No. & $\%$ & & \\
\hline Reflexes: & & & & & & \\
\hline Suckling & 57 & 50.9 & 67 & 59.8 & 1.81 & 0.18 \\
\hline Moro & 78 & 69.6 & 53 & 47.3 & 11.49 & $0.001 *$ \\
\hline Grasp & 12 & 10.7 & 5 & 4.5 & 3.12 & 0.08 \\
\hline Scalp molding & 4 & 3.6 & 16 & 14.3 & 7.91 & $0.005^{*}$ \\
\hline Caput succedaneum & 3 & 2.7 & 80 & 71.4 & 113.48 & $<0.001 *$ \\
\hline Eyes: & & & & & & \\
\hline Equal size & 104 & 92.9 & 87 & 75.9 & & \\
\hline Unequal size & 8 & 7.1 & 27 & 24.1 & 12.22 & $<0.001 *$ \\
\hline Nasal obstruction & 2 & 1.8 & 25 & 22.3 & 22.28 & $<0.001 *$ \\
\hline Asymmetric thorax: & 22 & 19.6 & 60 & 53.6 & 27.78 & $<0.001^{*}$ \\
\hline $\begin{array}{l}\text { Extremities movement: } \\
\text { Normal }\end{array}$ & 100 & 89.3 & 83 & 74.1 & & \\
\hline Abnormal & 3 & 2.7 & 22 & 19.7 & 8.63 & $0.003 *$ \\
\hline Abdomen: & & & & & & \\
\hline Soft & 74 & 66.1 & 36 & 32.1 & & \\
\hline Firm & 36 & 32.1 & 49 & 43.8 & 36.67 & $<0.001 *$ \\
\hline Distended & 2 & 1.8 & 27 & 24.1 & & \\
\hline $\begin{array}{l}\text { Umbilical cord: } \\
\text { Moist }\end{array}$ & 81 & 72.3 & 32 & 28.6 & & \\
\hline Dry & 31 & 27.7 & 78 & 69.6 & -- & -- \\
\hline Foul & 0 & 0.0 & 2 & 1.8 & & \\
\hline Genitalia: & & & & & & \\
\hline Normal male & 51 & 45.5 & 58 & 51.8 & & \\
\hline Normal female & 59 & 52.7 & 46 & 41.1 & -- & -- \\
\hline Ambiguous & 2 & 1.8 & 8 & 7.1 & & \\
\hline Imperforate anus & 6 & 5.4 & 48 & 42.9 & 43.04 & $<0.001 *$ \\
\hline Breath sounds: & & & & & & \\
\hline $\begin{array}{l}\text { Equal } \\
\text { Unequal }\end{array}$ & $\begin{array}{l}81 \\
31\end{array}$ & $\begin{array}{l}72.3 \\
27.7\end{array}$ & $\begin{array}{l}25 \\
87\end{array}$ & $\begin{array}{l}22.3 \\
77.7\end{array}$ & 56.16 & $<0.001 *$ \\
\hline
\end{tabular}

(*) Statistically significant at $p<0.05$

(--) Test result not valid

Table (7): Newborn Apgar scores and NICU admission in the study and control groups

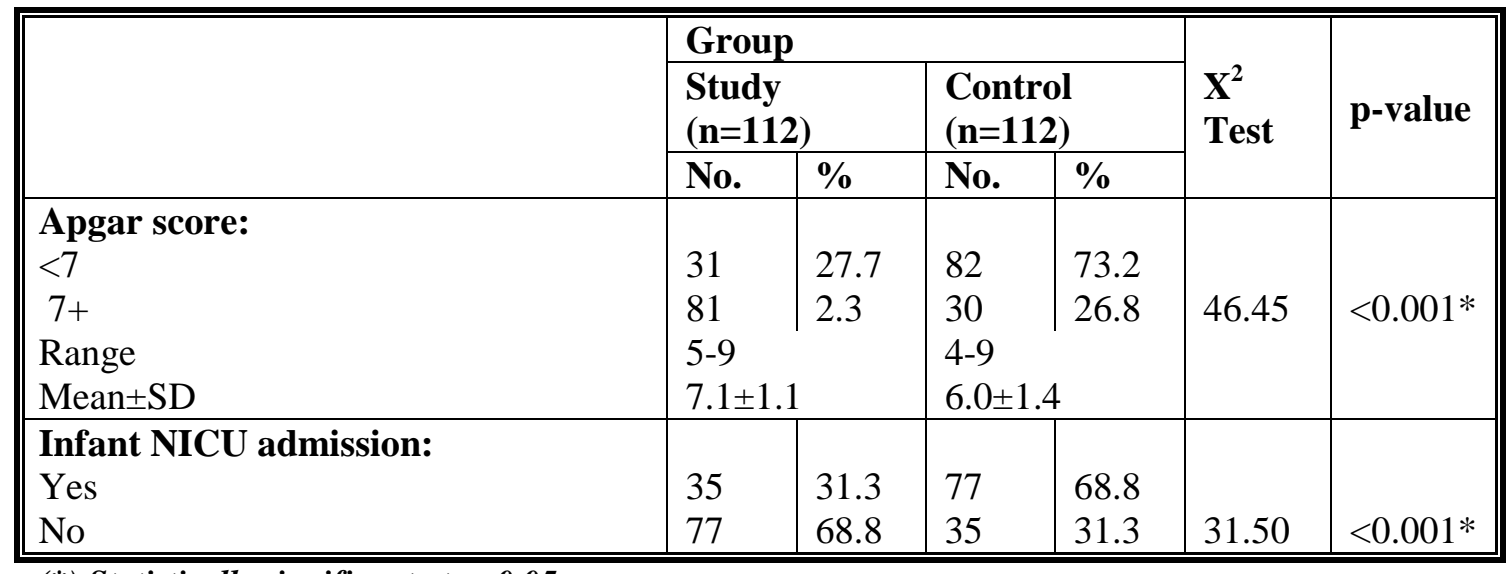

(*) Statistically significant at $p<0.05$ 
Table (8): Relation between post-intervention intake of balanced diet among women in the study group and their personal characteristics

\begin{tabular}{|c|c|c|c|c|c|c|}
\hline & \multicolumn{4}{|c|}{ Take balanced diet } & \multirow{3}{*}{$\begin{array}{l}X^{2} \\
\text { Test }\end{array}$} & \multirow{3}{*}{ p-value } \\
\hline & \multicolumn{2}{|l|}{ No } & \multicolumn{2}{|l|}{ Yes } & & \\
\hline & No. & $\%$ & No. & $\%$ & & \\
\hline $\begin{array}{l}\text { Age (years): } \\
<25 \\
25- \\
30-35\end{array}$ & $\begin{array}{l}24 \\
38 \\
29\end{array}$ & $\begin{array}{l}80.0 \\
84.4 \\
78.4\end{array}$ & $\begin{array}{l}6 \\
7 \\
8\end{array}$ & $\begin{array}{l}20.0 \\
15.6 \\
21.6\end{array}$ & 0.53 & 0.77 \\
\hline $\begin{array}{l}\text { Education: } \\
\text { Illiterate/read } \\
\text { Basic/intermediate } \\
\text { University }\end{array}$ & $\begin{array}{l}9 \\
53 \\
29\end{array}$ & $\begin{array}{l}90.0 \\
84.1 \\
74.4\end{array}$ & $\begin{array}{l}1 \\
10 \\
10\end{array}$ & $\begin{array}{l}10.0 \\
15.9 \\
25.6\end{array}$ & 2.06 & 0.36 \\
\hline \begin{tabular}{|l|} 
Job: \\
Housewife \\
Working \\
\end{tabular} & $\begin{array}{l}19 \\
72 \\
\end{array}$ & $\begin{array}{l}86.4 \\
80.0\end{array}$ & $\begin{array}{l}3 \\
18 \\
\end{array}$ & $\begin{array}{l}13.6 \\
20.0\end{array}$ & Fisher & 0.76 \\
\hline $\begin{array}{l}\text { Crowding index: } \\
<2 \\
2+\end{array}$ & $\begin{array}{l}74 \\
17\end{array}$ & $\begin{array}{l}81.3 \\
81.0\end{array}$ & $\begin{array}{l}17 \\
4\end{array}$ & $\begin{array}{l}18.7 \\
19.0\end{array}$ & Fisher & 1.00 \\
\hline $\begin{array}{l}\text { Husband age (years): } \\
<30 \\
30- \\
35+\end{array}$ & $\begin{array}{l}47 \\
25 \\
19\end{array}$ & $\begin{array}{l}82.5 \\
86.2 \\
73.1\end{array}$ & $\begin{array}{l}10 \\
4 \\
7\end{array}$ & $\begin{array}{l}17.5 \\
13.8 \\
26.9\end{array}$ & -- & -- \\
\hline $\begin{array}{l}\text { Husband job: } \\
\text { Employee } \\
\text { Worker } \\
\end{array}$ & $\begin{array}{l}62 \\
29 \\
\end{array}$ & $\begin{array}{r}79.5 \\
85.3 \\
\end{array}$ & $\begin{array}{l}16 \\
5\end{array}$ & $\begin{array}{l}20.5 \\
14.7\end{array}$ & 0.52 & 0.47 \\
\hline \begin{tabular}{|c|} 
Husband education: \\
Illiterate/read \\
Basic/intermediate \\
University \\
\end{tabular} & $\begin{array}{l}6 \\
59 \\
26\end{array}$ & $\begin{array}{l}100.0 \\
81.9 \\
76.5\end{array}$ & $\begin{array}{l}0 \\
13 \\
8 \\
\end{array}$ & $\begin{array}{l}0.0 \\
18.1 \\
23.5\end{array}$ & 1.92 & 0.38 \\
\hline \begin{tabular}{|l} 
Income: \\
Insufficient \\
Sufficient
\end{tabular} & $\begin{array}{l}63 \\
28 \\
\end{array}$ & $\begin{array}{l}84.0 \\
75.7\end{array}$ & $\begin{array}{l}12 \\
9 \\
\end{array}$ & $\begin{array}{l}16.0 \\
24.3 \\
\end{array}$ & 1.13 & 0.29 \\
\hline $\begin{array}{l}\text { In charge to buy food: } \\
\text { Woman herself } \\
\text { Other person }\end{array}$ & $\begin{array}{l}27 \\
64 \\
\end{array}$ & $\begin{array}{l}77.1 \\
83.1 \\
\end{array}$ & $\begin{array}{l}8 \\
13 \\
\end{array}$ & $\begin{array}{l}22.9 \\
16.9 \\
\end{array}$ & 0.56 & 0.45 \\
\hline $\begin{array}{l}\text { In charge to prepare food: } \\
\text { Woman herself } \\
\text { Other person }\end{array}$ & $\begin{array}{l}72 \\
19 \\
\end{array}$ & $\begin{array}{l}80.9 \\
82.6 \\
\end{array}$ & $\begin{array}{l}17 \\
4 \\
\end{array}$ & $\begin{array}{l}19.1 \\
17.4 \\
\end{array}$ & Fisher & 1.00 \\
\hline \begin{tabular}{|l|} 
Total knowledge: \\
xSatisfactory $(50 \%+)$ \\
Unsatisfactory $(<50 \%)$ \\
\end{tabular} & $\begin{array}{l}87 \\
4 \\
\end{array}$ & $\begin{array}{l}81.3 \\
80.0 \\
\end{array}$ & $\begin{array}{l}20 \\
1 \\
\end{array}$ & $\begin{array}{l}18.7 \\
20.0 \\
\end{array}$ & Fisher & 1.00 \\
\hline
\end{tabular}

(--) Test result not valid

\section{DISCUSSION}

Pregnancy is a powerful and a complex time in a woman's life. She may be happily looking forward to the birth of a long-awaited first child. She may be wondering how to make adjustments for another of many children. She may be waiting fearfully through the period of pregnancy (Simon et al., 2006; Towel, 2009). 
Family income was consider to be relevant difference in socio-demographic characteristics that was of statistical significance between women in the two groups. It was found that more women in the control group had sufficient income compared with the study group. This would have an influence on the availability to quality food in adequate amounts (Morland et al., 2002).

The present study showed increased trends of intake of balanced diet after intervention in the study group with women's educational level, working status, husband education, higher income, and being herself in charge of purchasing and preparing food. However, these trends lacked statistical significance because of the small numbers of women taking balanced diet, thus decreasing the statistical efficiency. Nevertheless, these trends are in agreement with previous studies that demonstrated the effect of socio-economic level (Groth et al., 2001; Dynesen et al., 2003; Mishra et al., 2005), higher educational levels (Freisling et al., 2006; Northstone et al., 2008)

As regards newborn outcomes, the present study revealed some significant differences between the study and control groups. Thus, the newborns in the study group had significantly better Apgar scores and lower rate of NICU admission. These positive fetal outcomes may be attributed to the better maternal nutritional status in this group, which is reflected on newborn's wellbeing. In congruence with this, Huh et al. (2005) showed that the dietary protein composition during pregnancy affects offspring vital signs.

Moreover, the newborns of women in the study group had significantly better anthropometric measurements as body weight and head circumference. These findings add to the evidence that the nutritional education program had a positive impact on maternal nutritional status, with subsequent positive effect on their newborns. In congruence with this, Yegammai et al (2002) studied the effect of nutrition education of mothers on nutritional status of infants at Coimbatore, India. It was observed that the infants born to the mothers with nutrition education recorded significantly higher birth weights than the infants born to the mothers without nutrition education. Similarly, Paul and Vijaylakshmi (2002) studied the effect of improving the maternal nutritional status on the birth weight of the newborn. The results revealed that mean birth weight of the neonates born to the mothers in control group was lower than those of the intervention groups.

Furthermore, a systematic review demonstrated that food-support programs may have a favorable effect on mean birth weight in high-risk sub-groups such as heavy smokers and very young mothers. However, none of the studies measured the effectiveness of the programs on mean birth weight in later pregnancies (D'Souza et al., 2006). Meanwhile, a study in India confirmed the positive relation between maternal nutritional status and birth weight of infants (Mridula et al., 2001). Also, Parvathi and Begum (2007) found that significantly more pregnant women with anemia gave birth to infants with very low birth weight and low birth babies. 
On the same line, Yegammai and Anuradha (2008) at Coimbatore, India observed that as maternal weight gain increased the weight of newborn was also increased. Also, Bang and Sunlee (2009) in Korea revealed a positive correlation between pregnant women's weight and their infants' birth weight. However, their study demonstrated that the maternal nutrient intake had greater influence on birth weight of infants than the maternal body weight. A similar relation between gestational weight gain and birth weight of infants was reported in a study in Belgium (Guelinckx et al., 2010).

In agreement with the present study finding regarding better head circumference values in the study group, Yegammai et al (2002) and Bhavana et al. (2004) demonstrated a positive association between maternal nutritional status and neonatal head circumference. This was also confirmed in a systematic review which concluded that it can be confidently said that programs can have a beneficial effect on newborn head circumference (D'Souza et al., 2006).

The current study has also demonstrated significantly better physical assessment findings in the study group newborns, compared to the control group. This was shown in vital signs as heart rate, better reflexes, less scalp molding and caput succedaneum which indicate prolonged labor, and less abnormal findings. While not all these findings may be attributed to maternal nutritional status, many of them do. These results are in agreement with previous studies which demonstrated that maternal diet during pregnancy may be related many aspects of newborn health such as the respiratory outcomes (Romieu et al., 2007; Chatzi and Kogevinas, 2009), neurodevelopment (Oken and Bellinger, 2008), and bone health (Yin et al., 2010).

\section{CONCLUSION:}

\section{Based on study findings, it can be concluded that:}

Educational program has a significant and improvement impact on the at pregnant women's knowledge and practice related to nutritional care based on scientific background.

\section{RECOMMENDATIONS:}

- The educational program which proved successful should be integrated in the ANC program at the study setting and in similar ones.

- Training programs are recommended for nurses in order to enhance their knowledge and skills regarding nutrition during pregnancy in order to be able to educate and counsel pregnant women.

- Simple illustrative booklets and pamphlets in Arabic language should be prepared and made available in antenatal care units addressing practical issues related to the nutritional needs during pregnancy.

\section{REFERENCES:}

Alam D.S. (2009): Prevention of Low Birth Weight From research to practice. Department of international center for diarrhoeal disease research, Bangladesh, 
Dhaka, Bangladesh, Estle Nutr Work Shoposer Pediatr Program; 36: 209-21. discussion $221-5,259-68$.

Bang S.W., and Sunlee S. (2009): The factors affecting Pregnancy outcomes in the Second trimester pregnant women. Nutr. Res. Pract.; 3(2): 134-140.

Bhavana K., Kiran G., Neera A., and Kamlesh S. (2004): Effect of Maternal Nutritional Supplementation on fetal Growth Parameters and Doppler flow velocity in growth Restricted fetuses. The Indian J. Nutr. Dietet.; 41:198-204..

Chatzi L., and Kogevinas M. (2009): Prenatal and childhood Mediterranean diet and the development of asthma and allergies in children. Public Health Nutr;12:1629-34.

D'Souza L., Renfrew M., McCormick F., Dyson L., Wright K., Henderson J., and Thomas J. (2006): Food-support programs for low-income and socially disadvantaged childbearing women in developed countries: Systematic review of the evidence. National Institute for Health and Clinical Excellence.

Dynesen A.W., Haraldsdottir J., Holm L., and Astrup A. (2003): Socio-demographic differences in dietary habits described by food frequency questions - results from Denmark. Eur J Clin Nutr.; 57:1586-1597.

Freisling H., Elmadfa I., and Gall I. (2006): The effect of socioeconomic status on dietary intake, physical activity and body mass index in Austrian pregnant women. $\mathbf{J}$ Hum Nutr Diet.; 19:437-445.

Gabbe S.G., Niebyle J.R., Simpons J.L., (2007): Obstetrics normal and problem pregnancies, 5th ed., China: churchill living stone Elsevier, pp.1051-1053.

Groth M.V., Fagt S., and Brondsted L. (2001): Social determinants of dietary habits in Denmark. Eur J Clin Nutr.; 55:959-966.

Guelinckx I., Devlieger R., Mullie P., and Vansant G. (2010): Effect of lifestyle intervention on dietary habits, Physical activity, and gestational weight gain in obese pregnant women: a randomized controlled trial. American J. Clin. Nutr.; 91: 373-380. 
Huh S.Y., Rifas-Shiman S.L., Kleinman K.P., Rich-Edwards J.W., Lipshultz S.E., and Gillman M.W. (2005): Maternal protein intake is not associated with infant blood pressure. Int J Epidemiol. ; 34(2): 378-384.

Macintosh J., and Mack D. (2000): An integrative review illuminates curricular applications of primary health care. Journal of nursing education; 5(80): 116 - 122 .

Mishra G., Ball K., Patterson A., Brown W., Hodge A., Dobson A. (2005): Sociodemographic inequalities in the diets of mid-aged Australian women. Eur J Clin Nutr.; 59:185-195.

Morland K., Wing S., Roux A.D., and Poole C. (2002): Neighborhood characteristics associated with the location of food stores and food service places. Am J Prev Med.; 22:23-29.

Mridula D., Mishra C. P., and Chakravertya A. (2002): Effect of Mother's Dietary intake on Birth Weight of Newborn. The Indian J. Nutr. Dietet.; 39: 327-333.

Northstone K., Emmett P., and Rogers I. (2008): Dietary patterns in pregnancy and associations with socio-demographic and lifestyle factors. European Journal of Clinical Nutrition.; 62:471-479.

Oken E., and Bellinger D.C. (2008): Fish consumption, methylmercury and child neurodevelopment. Curr Opin Pediatr;20:178-83.

Parvathi M., and Begaum K. (2007): Dietary intakes, Anthropometric measurements and Pregnancy outcome among women from low income families. The Indian J. Nutr. Dietet.; 44: 484-491.

Paul M., and Vijaylakshmi P. (2002): Effect of Improving the Maternal Nutritional Status with Reference to Zinc, Vitamin A and Iron on the Birth weight of the Newborn. The Indian J. Nutr. Dietet.; 39: 95-104.

Romieu I., Torrent M., and Garcia-Esteban R. (2007): Maternal fish intake during pregnancy and atopy and asthma in infancy. Clin Exp Allergy;37:518-25.

Simon C., Everitt H., And Kendrick T. (2006): Routine care during pregnancy, 2nd ed., Oxford University Press. 
Taylor C., Lillis C., and Lemon P. (2001): Fundamentals of nursing, the art and science of nursing care, 4th ed., New York, London: J.B. Lippincott Company, pp. 61-145.

Towel M. (2009): Maternal newborn nursing care. New Jersey: Pearson prentice Hall; pp. $215-220$

Villar J, Farnot U., Barros F. (2000): A randomized trial of psychosocial support during high-risk pregnancies. The Latin American Network for Perinatal and Reproductive Research. N Engl J Med; 327:1266..

Yegammi C., and Anuradha P. (2008): Pregnancy Outcomes of women's from different income levels. The Indian J. Nutr. Dietet.; 45: 357-365.

Yin J., Dwyer T., Riley M., Cochrane J., and Jones G. (2010): The association between maternal diet during pregnancy and bone mass of the children at age 16. Eur J Clin Nutr;64:131-7. 


\section{تـأثير برنـامج تـعليمي غذائي للسيدات الحـوامـل للمـرة الأولـي علي الحمـل}

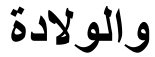

أ.د / سونية جمال الثرقاوى - أ.د/ حياة إمام محمد د/ ميرفت جابر زغلول- م.م / نور الهـى محمد السيد الثابورى أستاذ ورئيس قسم طب الاطفال ـ كلية الطب - جامعة قناة السويس ـ أستاذ تمريض صحة الأم وحديثي الولادة كلية التمريض ـ جامعة القاهرة ـ مدرس ، مدرس مساعد تمريض الأمومة و النساء و التوليذ ـ كلية التمريضجامعة بورسعيد الــــاصنـاصة

الدر اسة الحالية در اسة شبة تجريبية هدف الدر اسة هو تقييم تاثثر برنامج تعليمى عن التغذية اثناء الحمل على نتائج الحمل لدى الامهات البكريات. وشملت عينة الدراسة على مجمو عتين من 112امر اة حامل بكرية فى التلث الاول و الثانى من الحمل بمستشفى التضامن فى الفتره من شهر ابريل 2011 الى سبتمبر 2012 ، وقد تم تجميع البيانات عن طريق استخدام استمارة استبيان اشتملت على بيانات عن الخصائص الاجتماعية والديموغر افية بالاضافة الى تقييم المعرفة و العادات الغذائية استمارة فحص، قائمة ملاحظة نتائج الام. صممت الباحثة برنامجا تعليميا يهذف إلى تحسين معرفة المر أة الحامل و الممارسة المتعلقة بالر عاية التغذوية يعتمد على الخلفية العلمية ، وفي ضو اء الاحتياجات المحددة من التقييم القبلي. وتم تطبيق البرنامج على أعضاء مجمو عة الدراسة فقط ـ وقد تم تقييم المعرفة و الممارسات الغذائبة للمر أة بعد الو لادة في كل المجمو عتين. بالإضافة إلى ذللك، تمت مقارنة نتائج الحمل. وقد اسفرت النتائج على ان كانت المعرفة الغذائية قبل التدخل منخفضة في المجموعنين، مع عدم وجود فروق ذات دلالة إحصائية بينهما بينما أظهرت النتائج الاختبار البعدي أن 95.5٪ من مجموعة الدر اسة كن على معرفة مرضية مقارنة مع 18.8\% في المجمو عة الضابطة. كثفت النتائج عن تحسنات ذات دلالة إحصائية بعد التدخل في المعرفة و العادات الغذائية في مجموعة الدراسة ولكن ليس في المجمو عة الضابطة ـ الكلمات الإسترشادية :اهية التغذية السليمة اثثاء الحمل ناثثير التغذية السليمة على نتائج الحمل. 